\title{
The Immunogenicity of the Tumor-Associated Antigen $\alpha$-Fetoprotein Is Enhanced by a Fusion with a Transmembrane Domain
}

\author{
Lucile Tran,, ${ }^{1,}$ Jean-Paul Judor, ${ }^{1}$ Vanessa Gauttier, ${ }^{1}$ Michel Geist, ${ }^{2}$ \\ Chantal Hoffman, ${ }^{2}$ Ronald Rooke, ${ }^{2}$ Georges Vassaux, ${ }^{1}$ and Sophie Conchon ${ }^{1}$ \\ ${ }^{1}$ INSERM U948, Biothérapies Hépatiques, CHU Hotel Dieu, 44093 Nantes Cedex, France \\ ${ }^{2}$ Transgene SA, Boulevard Gonthier d'Andernach, 67405 Illkirch Graffenstaden, France \\ Correspondence should be addressed to Sophie Conchon, sophie.conchon@inserm.fr
}

Received 11 October 2011; Revised 10 November 2011; Accepted 10 November 2011

Academic Editor: Abdel A. Abdel-Rahman

Copyright () 2012 Lucile Tran et al. This is an open access article distributed under the Creative Commons Attribution License, which permits unrestricted use, distribution, and reproduction in any medium, provided the original work is properly cited.

\begin{abstract}
Aim. To investigate the ability of recombinant modified vaccinia virus Ankara (rMVA) vector to induce an immune response against a well-tolerated self-antigen. Methods. rMVA vectors expressing different form of $\alpha$-fetoprotein (AFP) were produced and characterized. Naïve mice were vaccinated with MVA vectors expressing the AFP antigen in either a secreted, or a membranebound, or an intracellular form. The immune response was monitored by an IFN $\gamma$ ELISpot assay and antibody detection. Results. Vaccination with the membrane-associated form of AFP induced a stronger CD8 ${ }^{+}$T-cell response compared to the ones obtained with the MVA encoding the secreted or the intracellular forms of AFP. Moreover, the vaccination with the membrane-bound AFP elicited the production of AFP-specific antibodies. Conclusions. The AFP transmembrane form is more immunogenic. Expressing a membrane-bound form in the context of an MVA vaccination could enhance the immunogenicity of a self-antigen.
\end{abstract}

\section{Introduction}

Hepatocellular carcinoma (HCC) is one of the most common malignancies in the world (700, 000 deaths/year) and its incidence is increasing [1]. Therapeutic options available include liver transplantation, surgical resection and local ablative therapies, but the prognosis of HCC remains poor due to high tumor recurrence rates [2]. Thus, new strategies are needed and immunotherapy based on stimulation of the antitumor immune response is a promising approach [3].

AFP is a single-chain polypeptide belonging to the albumin family. Secreted AFP, synthesized in the yolk sac, the fetal liver, and the gastrointestinal tract, is the main serum protein during the fetal development [4]. Its expression is repressed shortly after birth and replaced by serum albumin. However, AFP is reexpressed in 50 to $80 \%$ of HCC, whereas its expression is low or absent in normal adult liver. Serum AFP is used as tumor marker for HCC diagnosis and followup.
Several vaccination strategies based on AFP as a TAA have been investigated for HCC immunotherapy. Briefly, AFP can be presented as an antigen by human and murine DC and persisting $\mathrm{T} \mathrm{CD}^{+}$memory clones present in the human and the murine repertories can recognize some peptide epitopes derived from AFP [5-8]. In murine models, murine AFP can function as a tumor rejection antigen in melanoma models [9] and AFP-specific vaccination can significantly impair the growth of an autochthonous hepatocellular carcinoma [10]. In the two conducted clinical trials, AFP-specific CTL have been detected in blood after AFP peptides administration but, however, it has not been correlated to an objective clinical response $[7,8]$. This could be due to the fact that onco-fetal antigens, including AFP, are self-tolerated molecules and that the tumor immune evasion mechanisms have to be successfully controlled to trigger an efficient antitumor response. Vaccination with altered forms of the antigen or with the xenogeneic protein is a possible strategy to overcome the tolerance to antigens [11-13]. 
Modified vaccinia virus Ankara (MVA) is a highly attenuated vaccinia virus [14]. It has been successfully used to raise immune response against various antigens $[15,16]$. Compared to immunotherapy strategies with monoclonal antibodies or with immunodominant peptides, vaccination with MVA encoding a tumor antigen presents the advantage of raising an immune response against the whole antigen. In the context of a MVA vaccination, the primary induction of the CTL response depends mostly on cross-presentation suggesting that the expression of a full-length antigen localized subcellularly should be considered during the MVA vector design [17].

In the present study, we compare the immune response elicited by the vaccination with a recombinant MVA vector encoding the AFP antigen either in its native secreted form or in modified membrane-associated or intracellular forms.

\section{Materials and Methods}

2.1. Cell Culture. Primary chicken embryo fibroblasts (CEF) were used for homologous recombination and amplification of recombinant vectors. CEF were prepared from chicken embryos obtained from fertilized eggs (Lohmann, France) previously incubated 11 days at $37^{\circ} \mathrm{C}$ in a humid atmosphere. Embryos were dissected and treated with a TripLE Select solution (Invitrogen) (w/v). CEF cells were maintained in Eagle-based medium supplemented with $5 \%$ fetal bovine serum.

2.2. Plasmid Constructions and MVA Recombinants Production. The MVA shuttle plasmid contains a multiple-cloning site under the control of the $\mathrm{pH} 5 \mathrm{R}$ early-late vaccinia virus promoter surrounded by the flanking sequences of the deletion III of the MVA allowing homologous recombination and a selection gene, the E. coli xanthine-guanine phosphoribosyl-transferase gene (GPT gene) under the control of the $\mathrm{p} 11 \mathrm{~K} 7.5$ promoter [18].

Full-length cDNA encoding the secreted form of murine AFP (S-mAFP) was obtained by PCR (primers F5'-GGCCGCGCTAGCCGCCACCATGAAGTGGATCACACCCGCTTC-3'; R5'-GCAGTCAGGGCCCCTGCACTCAGTAATACATAAACGCCCAAAGCATCACGAGTTTTG$\left.3^{\prime}\right)$ and digestion with NheI and NotI enzymes (New England Biolabs) on pCIneomAFP [19]. The intracellular AFP sequence (I-mAFP) was obtained by deleting the exporting signal (nucleotides 1 to 54) by PCR (Primers F5'-GGCCGCGCTAGCCGCCACCATGAAAGCATTGCACGAAAATGAGTT-3'; R5'-GAGCCACATCCAGGGCCAGCTTC$\left.3^{\prime}\right)$ and enzyme digestion. The transmembrane AFP (TmAFP) sequence was obtained by successive PCR and fusion between the S-mAFP sequence and a rabies transmembrane domain, kindly provided by Dr. N. Silvestre (Transgene). The resulting sequences, verified by sequencing, are described in Figure 1(a) and were cloned into the corresponding sites of the MVA shuttle plasmid.

The generation of recombinant MVAs was previously described in detail [20]. Briefly, CEF cells were infected with a MVA without any inserted transgene (MVA-null) and then transfected with the different shuttle plasmids by
$\mathrm{CaCl}_{2}$ precipitation. Homologous recombination occurred and recombinant MVA viruses are isolated by multiple steps of mycophenolic acid selection. Finally, the selection marker is eliminated by multiple passages without selection. Final recombinant MVA viruses were controlled by PCR, amplified in CEF, and virus stocks were titrated on CEF by plaque assay.

2.3. Western Blot Analysis. CEF cells were infected with each MVA vector at a multiplicity of infection (MOI) of 0.2 and incubated for $48 \mathrm{~h}$. Cell lysate proteins were run on a $10 \%$ SDS-PAGE gel under reducing conditions and transferred onto a nitrocellulose membrane. The membrane was incubated with a polyclonal goat anti-AFP (C-19) antibody (Santa Cruz) at a 1:1000 dilution or with a rabbit antirabies transmembrane domain at a 1:2000 dilution. The membrane was then washed and incubated with a secondary antibody coupled with horseradish peroxidase (GE Healthcare). Signal detection was done by enhanced chemiluminescence (GE Healthcare).

2.4. Animal Experiments. $\mathrm{C} 3 \mathrm{H} / \mathrm{HeN}(\mathrm{C} 3 \mathrm{H})$ male mice $(8$ weeks) were purchased from Janvier Laboratory (France) and were bred in the Nantes IFR 26 animal facility. All experiments procedures involving animals were conducted according to the guidelines of the French Agriculture Ministry and were approved by the local ethical committee. Mice were immunized three times subcutaneously (s.c.) into the flank with $5 \cdot 10^{7} \mathrm{pfu}$ of MVA vectors at 7-day intervals. Spleen cells from immunized mice were harvested 1 week after the last boost.

Mice subjected to regulatory $\mathrm{T}$ cells depletion were injected intraperitoneally (i.p.) 1 day prior the vaccination course with $400 \mu \mathrm{g}$ of anti-CD25 antibody (PC61) purchased from BioXCell (New Hampshire, USA).

2.5. Enzyme-Linked Immunospot Analysis. Total splenocytes were extracted mechanically from spleen at sacrifice. $\mathrm{CD} 8^{+} \mathrm{T}$ cells were isolated from spleen cells with mouse CD8a (Ly2) Microbeads and MS columns (Miltenyi Biotec). Specific T-cell response was monitored on $\mathrm{CD}^{+} \mathrm{T}$ cells with IFN $\gamma$ ELISpot kit (Diaclone), according to the manufacturer's protocol. Cells, plated at a concentration of $5 \times 10^{5}$ cells per well, were stimulated with $20 \mu \mathrm{M}$ of a mAFP-specific CD $8^{+}$ restricted peptide (NEFGIASTL) described earlier [19], with medium alone or with MVA-null at MOI 10 for $24 \mathrm{~h}$. IFN $\gamma$ secreting cells were counted on an AID ELISpot Reader 9 (Autoimmun Diagnostika). Results are presented as the number of IFN $\gamma$-secreting cells per million of $\mathrm{CD}^{+} \mathrm{T}$ cells after subtraction of nonspecific signal obtained with medium alone.

2.6. Antibody Response. Blood samples were collected on days $0,7,14$, and 21 during the vaccination course. Plasma was separated by centrifugation from blood collected. A fusion protein consisting of the native murine AFP and a V 5 tag was used in ELISA to detect anti-AFP serological responses. AFP open reading frame was inserted in frame with a sequence encoding the V5 epitope in the pcDNA3.1/V5His vector (Invitrogen). 293 cells were transfected with the 


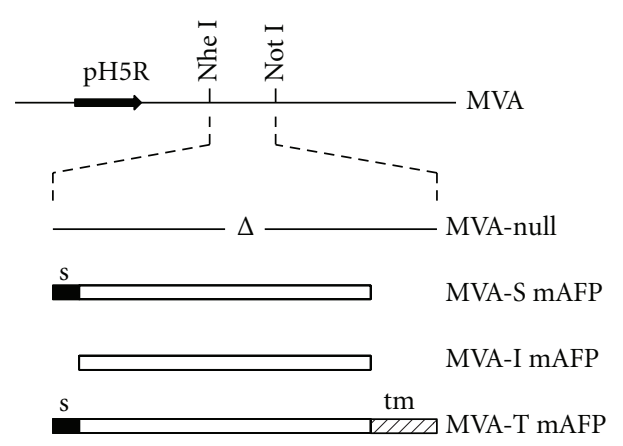

(a)

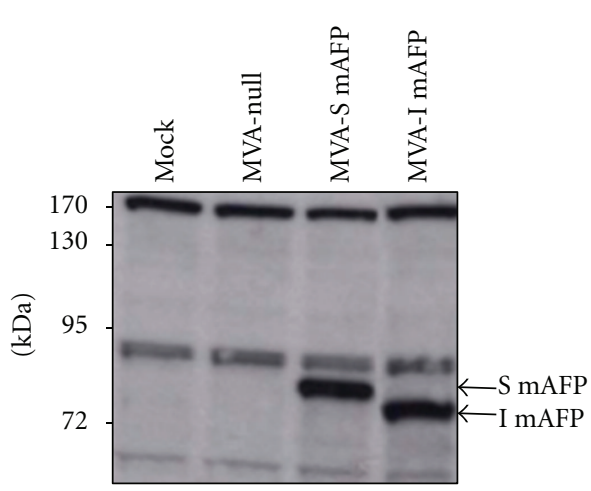

(b)

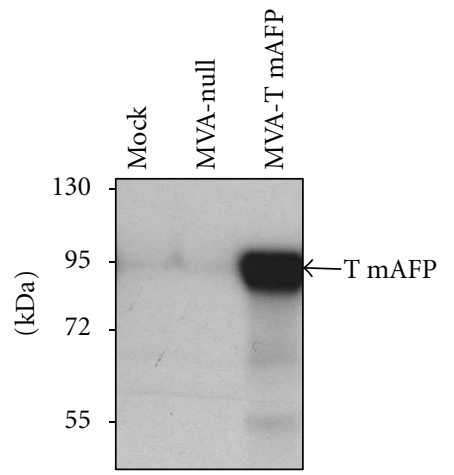

(c)

FIGURE 1: Generation of recombinants MVA expressing the different murine AFP sequences and evaluation of the mAFP proteins expression. (a) Schematic representation of transgenes inserted into the MVA genome. The different AFP sequences are under the control of the earlylate promoter pH5R. $\Delta$ : no insert; s: exporting sequence; tm: transmembrane domain; (b) specific detection of modified AFP proteins on western blot by polyclonal antibody directed against the C-terminal AFP protein. Lane 1 (left to right): mock-infected CEP cells; Lane 2: CEP cells infected with MVA-null; Lane 3: CEP cells infected with MVA-s mAFP; Lane 4: CEP cells infected with MVA-I mAFP. Molecular weight standards are shown in $\mathrm{kDa}$ on the left. The presence of the intracellular AFP protein (I mAFP) and of the secreted AFP protein (S mAFP) is indicated (arrows). (c) Specific detection of transmembrane AFP protein on western blot by antibody directed against the transmembrane domain. The presence of the transmembrane AFP protein (T mAFP) is indicated (arrow).

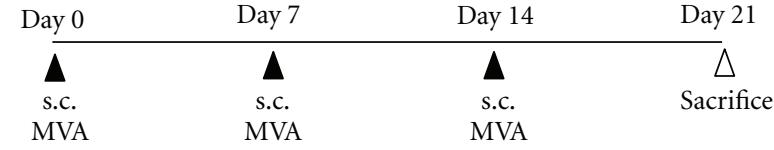

(a)

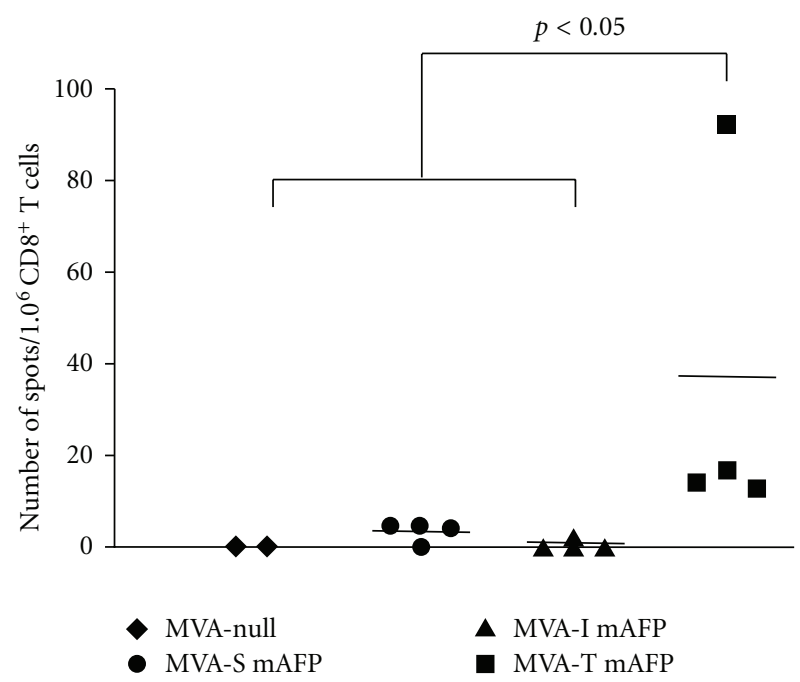

(b)

FIgURE 2: AFP-specific CD8 ${ }^{+}$T-cell immune response after vaccination of C3H/HeN mice with MVA-s mAFP, MVA-I mAFP, or MVA-T mAFP. (a) Schematic of the experimental design; (b) IFN $\gamma$ ELISpot assay, CD8 ${ }^{+}$T cells from mice vaccinated with MVA-null ( $\checkmark$ : negative control) or MVA expressing the secreted $(\bullet)$, the intracellular $(\mathbf{\Delta})$, or the transmembrane $(\mathbf{\square})$ form of murine AFP protein were stimulated in vitro by a specific AFP peptide. Statistical analyses were performed by the Kruskal-Wallis test.

resulting plasmid with Lipofectamine (Invitrogen) and the AFP-V5 fusion protein was secreted in the culture supernatant. 96-well plates were coated overnight at $4^{\circ} \mathrm{C}$ with a goat anti-V5 tag antibody (Abcam, UK) at $1 \mu \mathrm{g} / \mathrm{mL}$ diluted in bicarbonate coating buffer. Following blocking with PBS-Tween-milk (5\%) (PTM), plates were incubated overnight at $4^{\circ} \mathrm{C}$ with $100 \mu \mathrm{L}$ of supernatant containing the AFP-V5 fusion protein. Wells were then washed three times in PTM and incubated overnight at $4^{\circ} \mathrm{C}$ with primary mouse sera diluted serially in PTM. Wells were then washed three times in PTM and incubated with a goat anti-mouse IgG HRP secondary antibody (DAKO) for $2 \mathrm{~h}$ at room temperature. Following washings in PTM, wells were incubated with an ABTS substrate (Roche Diagnostics, 


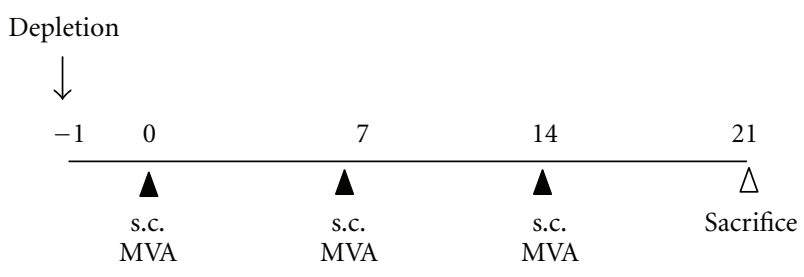

(a)

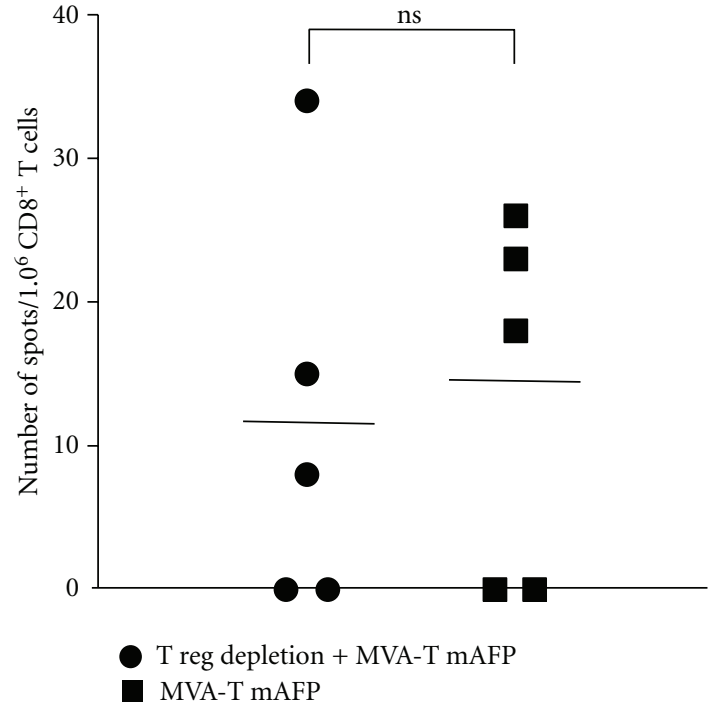

(b)

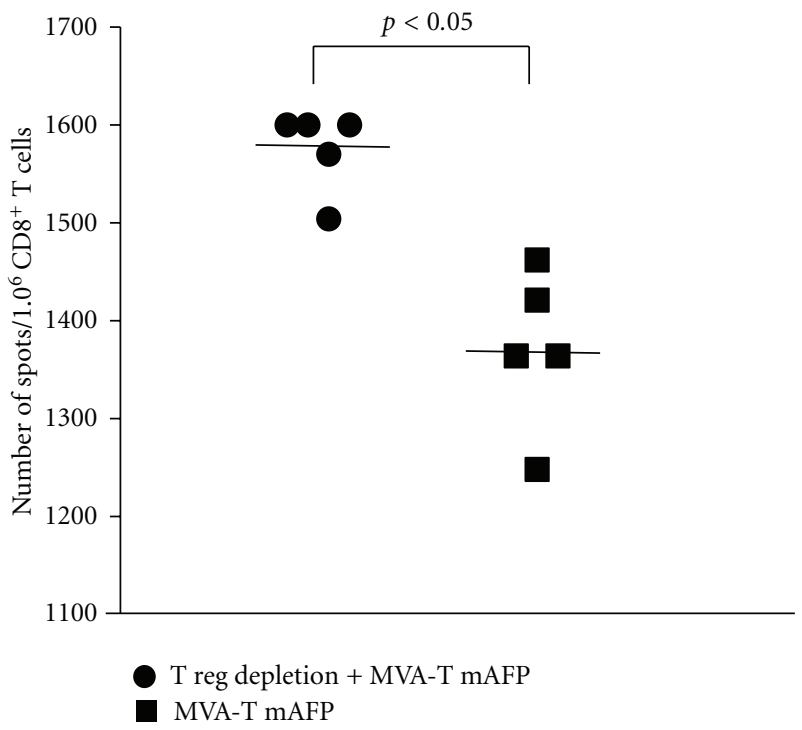

(c)

FIgURE 3: Effect of T reg depletion on AFP-specific and MVA-specific CD8 ${ }^{+}$T-cell immune response. (a) Schematic of the experimental design. (b) AFP-specific CD8 ${ }^{+} \mathrm{T}$ cells from MVA-T mAFP vaccinated mice in combination with $\mathrm{T}$ reg depletion $(\mathbf{\bullet})$ or not $(\mathbf{\square})$ were measured by IFNg ELISpot assay. (c) MVA-specific CD8 ${ }^{+} \mathrm{T}$ cells from MVA-T mAFP vaccinated mice in combination with T reg depletion (-) or not ( $)$ were measured by IFNg ELISpot assay after in vitro stimulation with the MVA-null vector. Statistical analyses were performed by the Mann-Whitney test.

Germany). The colorimetric change was monitored using a spectrophotometer.

2.7. Statistical Analysis. Statistical analyses were performed using the non-parametric Kruskal-Wallis or Mann-Whitney tests and GraphPad Prism software. A $P$ value $<0.05$ was considered to be statistically significant.

\section{Results}

3.1. Generation of Recombinants MVA Expressing AFP. The sequences corresponding to the secreted ( $\mathrm{mAFP}$ ), intra- cellular (I mAFP), and transmembrane ( $\mathrm{T}$ mAFP) forms of the murine AFP protein were introduced in the deletion III of the MVA genome under the control of the early-late promoter $\mathrm{pH} 5 \mathrm{R}$. The recombinants MVA produced are presented in Figure 1(a). Expression of the modified AFP proteins was confirmed by western blot. Specific signals corresponding to the intracellular and to the secreted mAFP proteins in cellular extracts isolated from CEP cells infected either by MVA-I mAFP (Figure 1(b), lane 1) or by MVA-s mAFP (Figure 1(b), lane 2) were detecting using a polyclonal antibody directed against the C-terminal part of the AFP protein. The respective sizes of these two proteins are in 


\begin{tabular}{|c|c|c|c|}
\hline \multicolumn{4}{|c|}{ Blood collect } \\
\hline$\Gamma$ & $T$ & $T$ & ᄀ \\
\hline Day 0 & Day 7 & Day 14 & Day 21 \\
\hline$\Delta$ & $\Delta$ & $\Delta$ & $\triangle$ \\
\hline s.c. & s.c. & s.c. & Sacrifice \\
\hline MVA & MVA & MVA & \\
\hline
\end{tabular}

(a)

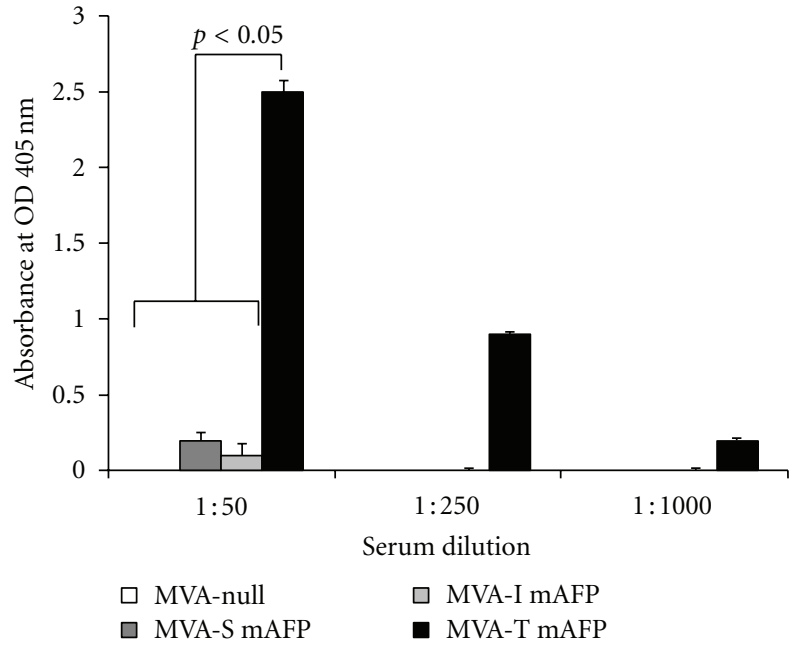

(b)

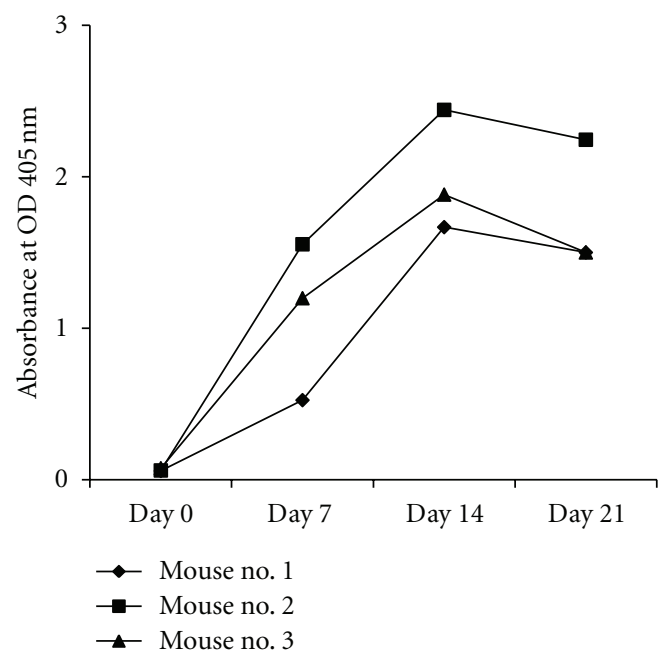

(c)

FIgURE 4: Induction of AFP-specific antibodies after vaccination with MVA-T mAFP. (a) Schematic of the experimental design. (b) ELISA analysis of sera from mice vaccinated with MVA-null, MVA-s mAFP, MVA-I mAFP, or MVA-T mAFP. Mean absorbances are determined at dilutions $1: 50,1: 250$, and $1: 1000$. (c) Time course of AFP-specific antibodies titre in sera from mice $(n=3)$ vaccinated with MVA-T mAFP. The absorbance is determined for a $1: 50$ dilution.

line with the expected size of the native mAFP protein and with the molecular modifications achieved. As the antibody previously used was directed against the C-terminal part of the protein where the fusion was made, the expression of the transmembrane form was confirmed by western blot using an antibody directed against the transmembrane rabies domain. A specific protein band was detected at a higher size compared to the native protein in agreement to the fusion with the supplementary domain (Figure 1(c), lane 1).

\subsection{AFP-Specific CD8 ${ }^{+} T$ Lymphocyte Response to MVA-T} $m A F P, M V A-s$ mAFP, or MVA-I mAFP in Mice. Mice $(n=4)$ were vaccinated with the different recombinants MVA by three subcutaneous injections one week apart (Figure 2(b)). One week after the last immunization, the specific $\mathrm{CD}^{+}$ $\mathrm{T}$-cell response was measured in an interferon gamma
ELISpot assay, with the $\mathrm{H}-2^{\mathrm{k}}$ AFP peptide NEFGIASTL, as described previously [19]. As shown in Figure 2(b) and as expected, no specific response was detected in mice vaccinated with the parental vector MVA-null. Surprisingly, no significant increase in the number of spots was observed in mice vaccinated either with MVA-s mAFP or with MVA-I mAFP. In contrast, MVA-T mAFP-vaccinated mice displayed a significantly higher number of IFN- $\gamma$-producing cells compared to mice vaccinated with the other MVA vectors. Thus, in the context of AFP vaccination by an MVA vector, the transmembrane form elicits a higher $\mathrm{CD}^{+} \mathrm{T}$-cell immune response in $\mathrm{C} 3 \mathrm{H} / \mathrm{HeN}$-vaccinated mice.

\subsection{The AFP-Specific Immune Response Is Not Modulated by the CD25+ $T$ Cells. Populations of regulatory $\mathrm{T}$ cells could impair the immune response against a self-antigen such as}


AFP [21]. To address this possibility, groups of mice were vaccinated with the MVA-T mAFP vector, with or without an injection of $400 \mu \mathrm{g}$ of PC61 anti-CD25 monoclonal antibody the day before the first vaccination (Figure 3(a)). At sacrifice, $\mathrm{CD}^{+}{ }^{+} \mathrm{CD} 25^{+}$T-cell depletion was verified by FACS analysis (data not shown) and the $\mathrm{CD}^{+} \mathrm{T}$-cell response was compared in splenocytes prepared from the two groups by IFNg ELISpot assay. A single injection of anti-CD25 antibody did not enhance significantly the AFP-specific $\mathrm{CD}^{+} \mathrm{T}$ cell immune response (Figure $3(\mathrm{~b})$ ). In contrast, the $\mathrm{T}$ reg depletion has an impact on the immune response against the vector (Figure 3(c)). In fact, the antibody injection resulted in a significantly higher number of IFN- $\gamma$-producing $\mathrm{CD}^{+}$ T cells after MVA restimulation confirming that the depletion did occur.

3.4. Vaccination with MVA-T mAFP Elicits the Production of Anti-AFP Antibodies. To monitor the AFP-specific humoral response elicited by the vaccinations with the MVA recombinant vectors, mice $(n=3)$ were vaccinated either with the MVA AFP viruses or with the MVA-null, by three injections one week apart and sacrificed one week after. Sera were collected prior to each vaccination and at sacrifice. The serological response was assessed by ELISA as described in the Material and Methods section. The presence at sacrifice of circulating antibodies directed against the AFP protein was analyzed in mice vaccinated with the four different MVAs (Figure 4(b)). No AFP-specific antibody could be detected in the sera of mice vaccinated with MVA-null, or MVA$s$ mAFP or MVA-I mAFP, even at the minimal dilution ( $1: 50)$. In contrast, vaccination with MVA-T mAFP elicited a significantly stronger specific antibody response. The time course of AFP-specific antibody titer was assessed during the vaccination protocol (Figure 4(c)). Repeated immunizations (at day 0 , day 7 , and day 14) with MVA-T mAFP led to the production of AFP-specific antibodies which was maximal by day 14 and remained stable at day 21 .

\section{Discussion}

Tolerance to tumor-associated antigen is profound, therefore strenuous immune modulation is required to overcome it and achieve meaningful response. In this paper, we assessed the ability of MVA to induce an immune response against the self-antigen $\alpha$-fetoprotein (AFP), and we compared the immunogenicity of different forms of this antigen after vaccination in naïve mice. Three vaccines were generated to encode recombinant AFP that was either secreted or membrane-bound or cytosolic. Comparable strategies targeting antigen to specific subcellular compartments have been shown to efficiently modulate the specific immune response elicited [17]. The correct expression of the recombinant AFP forms by the MVA vectors was first confirmed. The vaccination protocol consisted in three subcutaneous injections one week apart and one week later, both the cellularspecific $\mathrm{CD}^{+} \mathrm{T}$-cell response and the humoral response were monitored. Expression of a cytosolic form of AFP failed to raise a strong immune response as what has been shown for a soluble cytosolic fragment of the Her-2 protein which is promptly degraded by the proteasome [22]. Vaccination with the transmembrane form of AFP induced a stronger $\mathrm{CD}^{+}{ }^{+} \mathrm{T}$-cell response compared to the ones obtained with the MVA encoding the secreted and the intracellular forms of AFP. Since the number of specific spots was limited, we evaluated the effect of a $\mathrm{T}$ reg depletion on the immune response obtained and we concluded that the AFP-specific $\mathrm{CD}^{+}$response was not modulated by the $\mathrm{CD}^{+} 5^{+} \mathrm{T}$ cell population during a MVA-T mAFP vaccination protocol. Finally, the vaccination with the transmembrane form of the antigen leads to the production of antibodies directed against AFP. Previously, vaccination with an MVA encoding a modified membrane-associated form of the epithelial tumor antigen $\mathrm{H} 23$, aberrantly expressed in breast cancer, has been shown to allow the production of elevated levels of specific antibodies in vaccinated animals $[23,24]$. The difficulty to raise a significant immune response against AFP compared to other TAA could be due to the fact that this self-antigen is produced by the liver, which is known to constitute a specific tolerogenic environment [25].

In conclusion, we demonstrate that the secreted and the intracellular AFP antigens expressed from MVA are less effective immunogens than their transmembrane counterpart. Vaccination with a membrane-bound form of a self-antigen, combined with other strategies, involving stimulation with cytokines (IL2, IL12, GM-CSF) [26, 27] could represent a promising strategy to enhance immunogenicity in the context of MVA vaccination.

\section{References}

[1] J. Ferlay, H. R. Shin, F. Bray, D. Forman, C. Mathers, and D. M. Parkin, "Estimates of worldwide burden of cancer in 2008: GLOBOCAN 2008," International Journal of Cancer, vol. 127, no. 12, pp. 2893-2917, 2010.

[2] J. Taieb, J. C. Barbare, T. Boussaha et al., "Management of hepatocellular carcinoma. Where are we now? What's next?" Bulletin du Cancer, vol. 96, no. 1, pp. 19-34, 2009.

[3] A. Zerbini, M. Pilli, C. Ferrari, and G. Missale, "Is there a role for immunotherapy in hepatocellular carcinoma?" Digestive and Liver Disease, vol. 38, no. 4, pp. 221-225, 2006.

[4] V. N. Evdokimova and L. H. Butterfield, " $\alpha$-Fetoprotein and other tumour-associated antigens for immunotherapy of hepatocellular cancer," Expert Opinion on Biological Therapy, vol. 8, no. 3, pp. 325-336, 2008.

[5] L. H. Butterfield, A. Koh, W. Meng et al., "Generation of human T-cell responses to an HLA-A2.1-restricted peptide epitope derived from $\alpha$-fetoprotein," Cancer Research, vol. 59, no. 13, pp. 3134-3142, 1999.

[6] L. H. Butterfield, W. S. Meng, A. Koh et al., "T cell responses to HLA-A*0201-restricted peptides derived from human $\alpha$ fetoprotein," The Journal of Immunology, vol. 166, no. 8, pp. 5300-5308, 2001.

[7] L. H. Butterfield, A. Ribas, V. B. Dissette et al., "A phase I/II trial testing immunization of hepatocellular carcinoma patients with dendritic cells pulsed with four $\alpha$-fetoprotein peptides," Clinical Cancer Research, vol. 12, no. 9, pp. 28172825, 2006.

[8] L. H. Butterfield, A. Ribas, W. S. Meng et al., "T-cell responses to HLA-A*0201 immunodominant peptides derived from $\alpha$ fetoprotein in patients with hepatocellular cancer," Clinical Cancer Research, vol. 9, no. 16, part 1, pp. 5902-5908, 2003. 
[9] C. M. Vollmer Jr., F. C. Eilber, L. H. Butterfield et al., “ $\alpha$ Fetoprotein-specific genetic immunotherapy for hepatocellular carcinoma," Cancer Research, vol. 59, no. 13, pp. 3064 3067, 1999.

[10] J. Cany, A. Avril, V. Pichard, D. Aubert, N. Ferry, and S. Conchon, "A transgenic mouse with $\beta$-Galactosidase as a fetal liver self-antigen for immunotherapy studies," Journal of Hepatology, vol. 47, no. 3, pp. 396-403, 2007.

[11] M. E. Engelhorn, J. A. Guevara-Patiño, T. Merghoub et al., "Mechanisms of immunization against cancer using chimeric antigens," Molecular Therapy, vol. 16, no. 4, pp. 773-781, 2008.

[12] E. Quaglino, C. Mastini, A. Amici et al., "A better immune reaction to Erbb-2 tumors is elicited in mice by DNA vaccines encoding rat/human chimeric proteins," Cancer Research, vol. 70, no. 7, pp. 2604-2612, 2010.

[13] J. C. Liao, P. Gregor, J. D. Wolchok et al., "Vaccination with human tyrosinase DNA induces antibody responses in dogs with advanced melanoma," Cancer Immunity, vol. 6, article 8, 2006.

[14] G. Sutter and B. Moss, "Nonreplicating vaccinia vector efficiently expresses recombinant genes," Proceedings of the National Academy of Sciences of the United States of America, vol. 89, no. 22, pp. 10847-10851, 1992.

[15] P. M. Arlen, J. L. Gulley, R. A. Madan, J. W. Hodge, and J. Schlom, "Preclinical and clinical studies of recombinant poxvirus vaccines for carcinoma therapy," Critical Reviews in Immunology, vol. 27, no. 5, pp. 451-462, 2007.

[16] B. Acres and J. Y. Bonnefoy, "Clinical development of MVAbased therapeutic cancer vaccines," Expert Review of Vaccines, vol. 7, no. 7, pp. 889-893, 2008.

[17] G. Gasteiger, W. Kastenmuller, R. Ljapoci, G. Sutter, and I. Drexler, "Cross-priming of cytotoxic $\mathrm{T}$ cells dictates antigen requisites for modified vaccinia virus Ankara vector vaccines," Journal of Virology, vol. 81, no. 21, pp. 11925-11936, 2007.

[18] F. G. Falkner and B. Moss, "Escherichia coli gpt gene provides dominant selection for vaccinia virus open reading frame expression vectors," Journal of Virology, vol. 62, no. 6, pp. 1849-1854, 1988.

[19] J. Cany, B. Barteau, L. Tran et al., "AFP-specific immunotherapy impairs growth of autochthonous hepatocellular carcinoma in mice," Journal of Hepatology, vol. 54, no. 1, pp. 115121, 2011.

[20] P. Erbs, A. Findeli, J. Kintz et al., "Modified vaccinia virus Ankara as a vector for suicide gene therapy," Cancer Gene Therapy, vol. 15, no. 1, pp. 18-28, 2008.

[21] K. Frimpong-Boateng, N. van Rooijen, and R. Geiben-Lynn, "Regulatory T cells suppress natural killer cells during plasmid DNA vaccination in mice, blunting the $\mathrm{CD}^{+} \mathrm{T}$ cell immune response by the cytokine TGF $\beta$," PLoS One, vol. 5, no. 8, Article ID e12281, 2010.

[22] S. A. Pilon, M. P. Piechocki, and W. Z. Wei, "Vaccination with cytoplasmic ErbB-2 DNA protects mice from mammary tumor growth without anti-ErbB-2 antibody," The Journal of Immunology, vol. 167, no. 6, pp. 3201-3206, 2001.

[23] M. Hareuveni, C. Gautier, M. P. Kieny, D. Wreschner, P. Chambon, and R. Lathe, "Vaccination against tumor cells expressing breast cancer epithelial tumor antigen," Proceedings of the National Academy of Sciences of the United States of America, vol. 87, no. 23, pp. 9498-9502, 1990.

[24] M. Hareuveni, D. H. Wreschner, M. P. Kieny et al., "Vaccinia recombinants expressing secreted and transmembrane forms of breast cancer-associated epithelial tumour antigen (ETA)," Vaccine, vol. 9, no. 9, pp. 618-626, 1991.
[25] V. Racanelli and B. Rehermann, "The liver as an immunological organ," Hepatology, vol. 43, no. 2, supplement 1, pp. S54S62, 2006.

[26] J. He, D. Shen, M. A. O’Donnell, and H. R. Chang, "Induction of MUC1-specific cellular immunity by a recombinant BCG expressing human MUC1 and secreting IL2," International Journal of Oncology, vol. 20, no. 6, pp. 1305-1311, 2002.

[27] J. Bubenik, "Genetically modified cellular vaccines for therapy of human papilloma virus type 16 (HPV 16)-associated tumours," Current Cancer Drug Targets, vol. 8, no. 3, pp. 180186, 2008. 


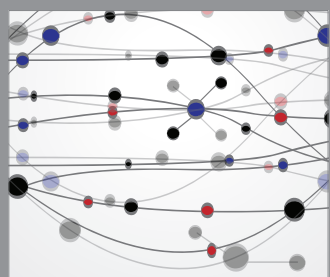

The Scientific World Journal
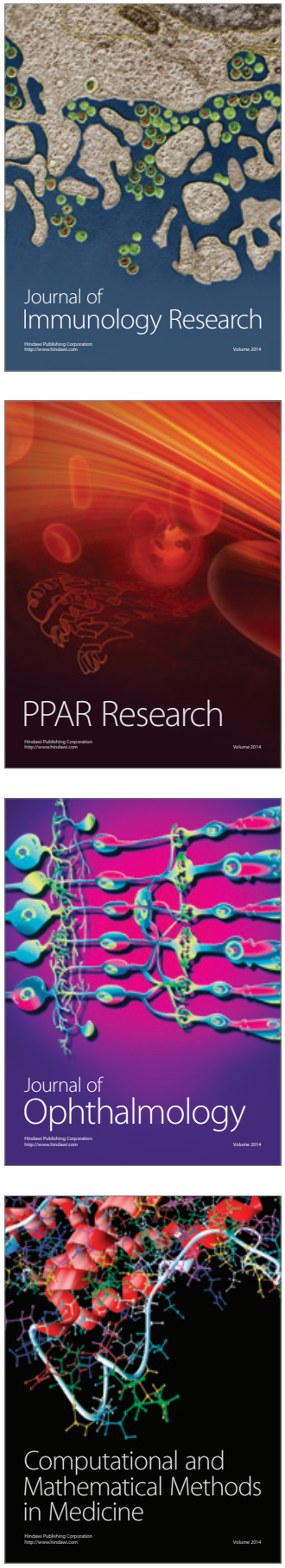

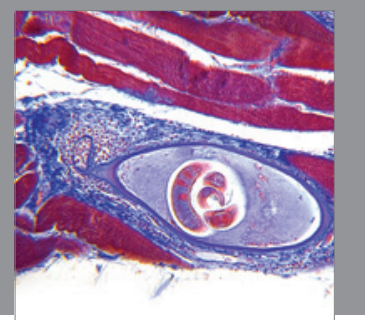

Gastroenterology

Research and Practice
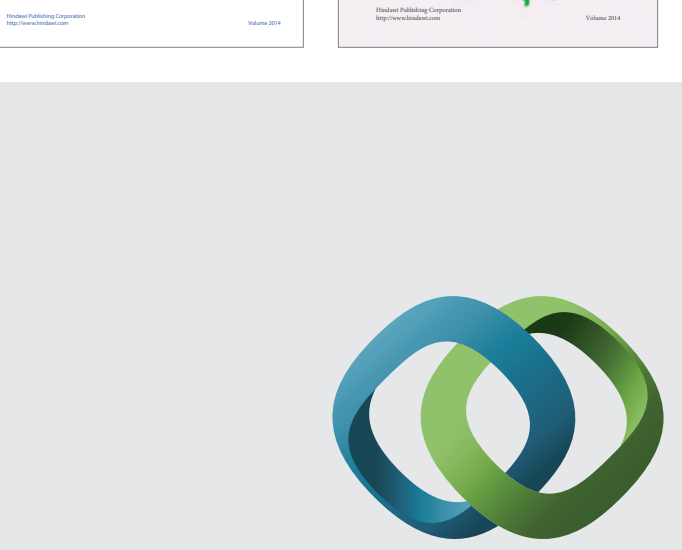

\section{Hindawi}

Submit your manuscripts at

http://www.hindawi.com
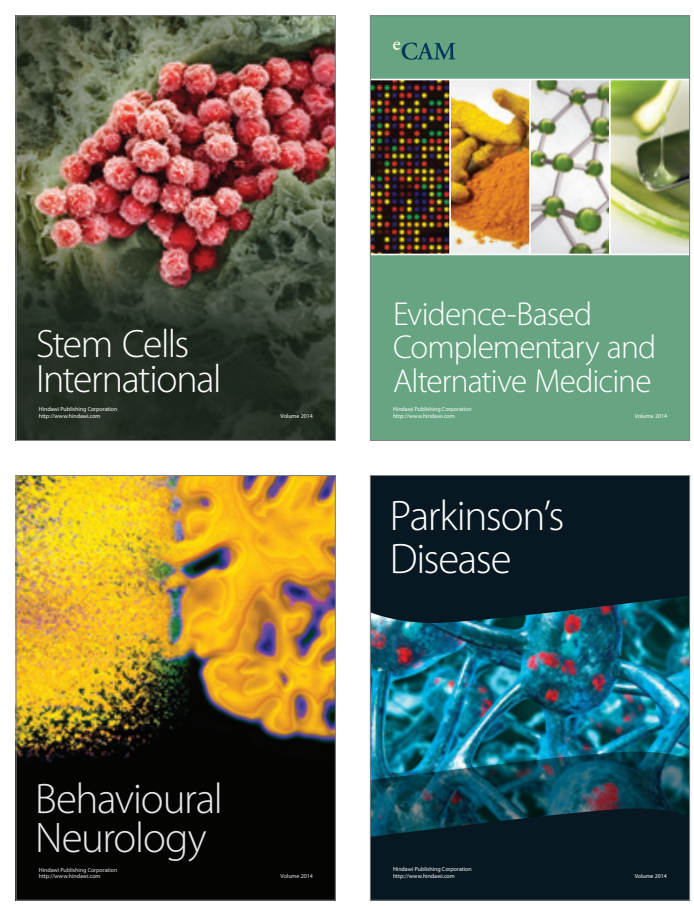

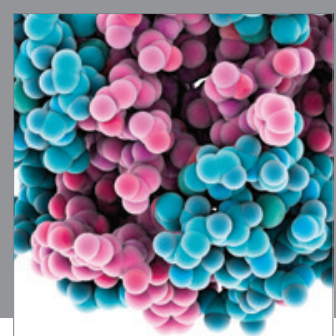

Journal of
Diabetes Research

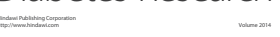

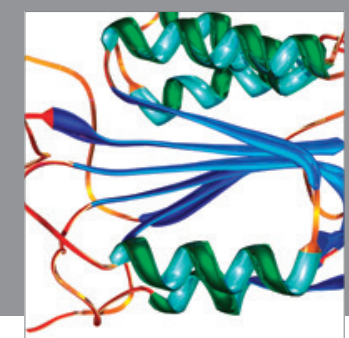

Disease Markers
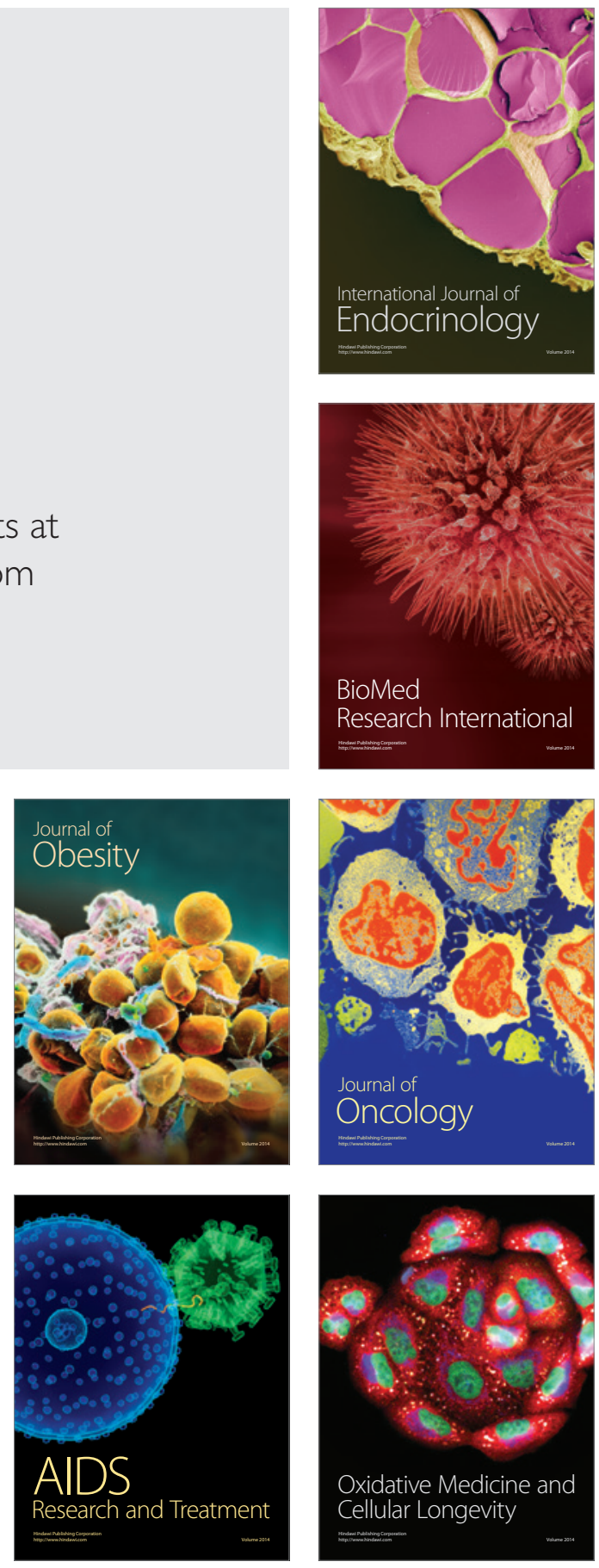